\title{
A Mutiparameter Joint Estimation Algorithm for Dual-Polarized Cylindrical Conformal Array in the Presence of Mutual Coupling
}

\author{
Chao Liu $(\mathbb{D}$ and Feiyang Zhou \\ Department of Electronics and Communications Engineering, Hefei University of Technology, Hefei 230601, China \\ Correspondence should be addressed to Chao Liu; disneyl@hfut.edu.cn
}

Received 7 June 2021; Revised 7 December 2021; Accepted 5 January 2022; Published 8 February 2022

Academic Editor: Francisco Falcone

Copyright (c) 2022 Chao Liu and Feiyang Zhou. This is an open access article distributed under the Creative Commons Attribution License, which permits unrestricted use, distribution, and reproduction in any medium, provided the original work is properly cited.

\begin{abstract}
In this article, we proposed a multiparameter joint estimation algorithm based on the dual-polarized cylindrical conformal array (DCCA) in the presence of mutual coupling. Using the characteristic of the dual-polarized cylindrical conformal array, 2D direction-of-arrival (DOA) estimation can be divided into 1D estimations of elevation and azimuth. Sensors on the boundary of the DCCA are set as auxiliary sensors to eliminate the influence of mutual coupling. Then, elevation can be estimated by the generalized eigenvalues utilizing signal subspace eigenvectors (GEESE). After that, polarization sensitivity can be eliminated by projection transformation and the proposed dual-polarized forward-backward smoothing algorithm. Consequently, a dualpolarized spatial spectrum can be developed to estimate the azimuth based on the estimated elevation. Furthermore, the angles of the signals can be reestimated to improve the accuracy of DOA estimation. Simulation results confirm the effectiveness of the proposed algorithm.
\end{abstract}

\section{Introduction}

Conformal arrays are widely used in aircraft, missiles, unmanned aerial vehicles, and other fields for the advantages of saving space, good aerodynamic characteristics, reducing radar cross-section, and increasing angular coverage [1]. However, due to the limited layout space of the conformal array, the electromagnetic coupling between the array elements becomes more serious [2]. And this effect will significantly degrade the performance of most existing DOA estimation methods. Common methods to eliminate the influence of mutual coupling are the iterative autocorrection method and auxiliary array element method [3]. In [4], the array is firstly divided into several subarrays, and then the MUSIC algorithm is used to realize the DOA estimation algorithm of the conformal array. However, the mutual coupling and polarization sensitivity of the array are not considered. In [5, 6], an iterative self-correction algorithm is proposed, which gradually obtains the optimal solution of angle and mutual coupling through loop iteration. This kind of multidimensional nonlinear solution algorithm has a large amount of calculation and is easy to fall into local optimum. In [7], an effective DOA estimation algorithm for the conformal array is proposed in the presence of mutual coupling. This algorithm reconstructs the steering vector of the subarrays and constructs a segmented space spectrum for DOA estimation. However, the influence of polarization sensitivity is not considered. In [8], a method is proposed to realize the joint estimation of mutual coupling and angle using an auxiliary element. However, the auxiliary element must be accurate and error-free, which is difficult to realize in many occasions.

Each polarized signal can be decomposed into two orthogonal components, and they can be modeled as a pair of coherent signals [9]. Thus, elimination of polarization sensitivity can also be seen as a process of decoherence. Common decoherence methods include spatial smoothing algorithm, signal feature vector method, and difference method [10]. Most of these methods are based on a specific array structure without universal applicability. In $[11,12]$, a DOA and polarization estimation algorithm for arbitrary array configurations is proposed. The algorithm separates 
the polarization sensitivity and angle information through the decoherence method to achieve DOA estimation. But the algorithm is not suitable for the scenario where mutual coupling and polarization sensitivity coexist. In [13], a blind DOA estimation algorithm is proposed for the conformal array. The algorithm uses a virtual transformation to avoid the effect of the polarization and the inconsistency of element pattern. Then, ESPRIT can be used to realize the DOA estimation. However, this method is based on the fourthorder cumulants of the array output, which brings a lot of calculation.

In DOA estimation of the conformal array, mutual coupling and polarization sensitivity still face great challenges. Scholars have proposed some DOA estimation algorithms for conformal array considering only polarization sensitivity or mutual coupling. These algorithms can achieve effective DOA estimation when only considering mutual coupling or polarization sensitivity, but they are no longer applicable with both of them. However, in the DOA of conformal array, mutual coupling and polarization sensitivity are inevitable factors. In view of this, we propose a mutiparameter joint estimation algorithm to solve this problem.

In this article, we use a method of stepped estimation to estimate the angle and polarization parameters. The $2 \mathrm{D}$ DOA estimation can be divided into two 1D estimations of elevation and azimuth according to the characteristic of DCCA. The influence of mutual coupling can be eliminated by setting the sensors on the boundary of the DCCA as auxiliary sensors. Elevation can be firstly estimated with the GEESE algorithm. Then, polarization sensitivity can be eliminated by projection transformation and dual-polarized forward-backward smoothing algorithm. After eliminating the influence of polarization sensitivity, we construct a spatial spectrum to estimate the azimuth. Consequently, polarization parameters can be estimated based on DOA estimation results. Since the polarization parameters have been obtained, the angles of the signals are reestimated to improve the accuracy of DOA estimation. Cramer-Rao bound (CRB) is also derived as a comparison standard of the estimation performance. Finally, Monte Carlo simulations are carried out to demonstrate the excellent performance of the proposed method.

\section{Signal Model}

Suppose a narrowband far-field signal with arbitrary polarization impinges on the array from direction $(\theta, \phi)$, where $\theta$ and $\phi$ denote the elevation and azimuth, respectively. Consider a dual-polarized cylindrical conformal array (DCCA) with $M \times N$ elements. The cylindrical array is spatially composed of $M$ layers, and each layer is a ring array with $N$ elements uniformly arranged. The distance between adjacent elements is $d_{x y}$, and the distance between adjacent layers is $d_{z}$, as shown in Figure 1.

\subsection{Dual-Polarized Cylindrical Conformal Array.} Considering the polarization inconsistency of the array elements, the polarization signal need to be decomposed and

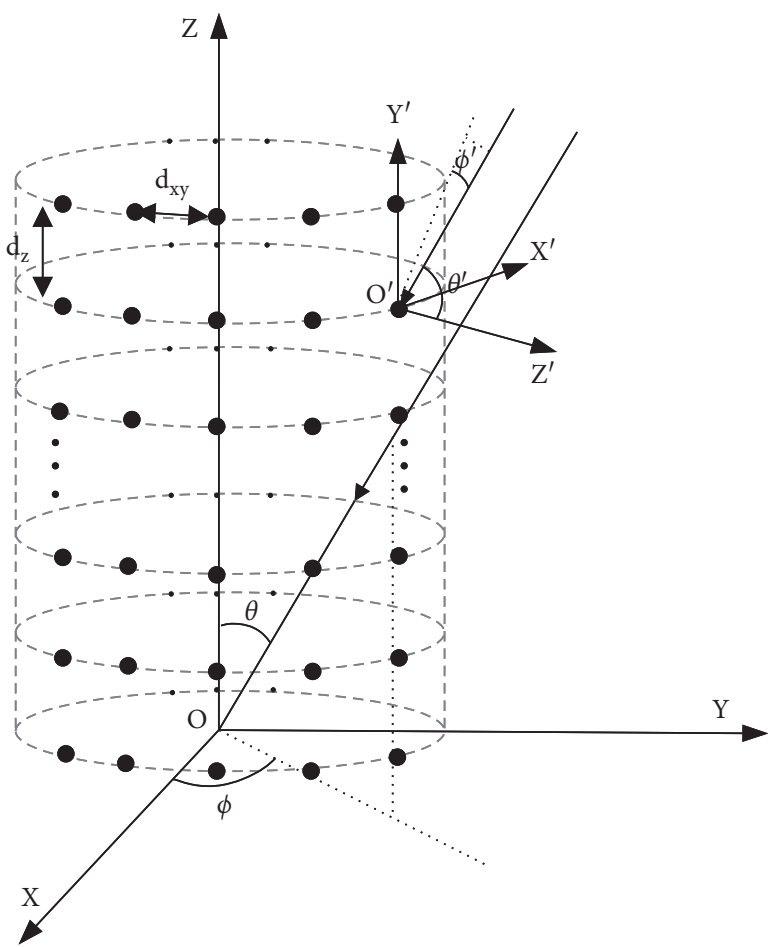

FIgURE 1: Conformal array coordinate diagram.

projected to the local coordinate system of each element to calculate the array response. Without considering the noise, the array output can be expressed as

$$
\begin{aligned}
\mathbf{x}(t) & =\mathbf{G}(\theta, \phi) \mathbf{T}(\theta, \phi)\left(\mathbf{a}(\theta, \phi) \otimes \mathbf{I}_{2}\right)\left[\begin{array}{l}
s_{\theta}(t) \\
s_{\phi}(t)
\end{array}\right], \\
\mathbf{G}(\theta, \phi) & {\left[\begin{array}{ccccc}
g_{\theta_{1}^{\prime}} & 0 & 0 & \ldots & 0 \\
0 & g_{\phi_{1}^{\prime}} & 0 & \ldots & 0 \\
0 & 0 & \ddots & 0 & 0 \\
\vdots & \vdots & 0 & g_{\theta_{M N}^{\prime}} & 0 \\
0 & 0 & \ldots & 0 & g_{\phi_{M N}^{\prime}}^{\prime}
\end{array}\right] } \\
\mathbf{T}(\theta, \phi) & =\left[\begin{array}{cccc}
\mathbf{T}_{1}(\theta, \phi) & \mathbf{0} & \ldots & \mathbf{0} \\
\mathbf{0} & \mathbf{T}_{2}(\theta, \phi) & \ldots & \mathbf{0} \\
\vdots & \vdots & \ddots & \vdots \\
\mathbf{0} & 0 & \ldots & \mathbf{T}_{M N}(\theta, \phi)
\end{array}\right] \\
\mathbf{a}(\theta, \phi) & {\left[a_{1}(\theta, \phi), \ldots, a_{M N}(\theta, \phi)\right]^{\mathrm{T}}, }
\end{aligned}
$$

where $g_{\theta_{n}^{\prime}}$ and $g_{\phi_{n}^{\prime}}(n=1,2, \ldots, M N)$ are patterns of $\theta$ polarization and $\phi$ polarization of the $n$th array element in the local coordinate system, respectively. $\mathbf{T}_{n}(\theta, \phi)$ represents transformation matrix from global coordinate system to local coordinate system of the $n$th array element [14]. $a_{n}(\theta, \phi)=e^{j k r_{n} \mathbf{v}}$, where $k=2 \pi / \lambda$ is the wavenumber, $\lambda$ is the wavelength of the signal, $\mathbf{r}_{n}=\left[x_{n}, y_{n}, z_{n}\right]$ is the position vector, and $\mathbf{v}=[\sin \theta \cos \phi \text {, } \sin \theta \sin \phi, \cos \theta]^{\mathrm{T}}$ is the unit direction vector. " $(\cdot)^{\mathrm{T}}$ " indicates the transpose of the matrix. $I_{2}$ represents a $2 \times 2$ identity matrix. “ $\otimes$ " represents the 
Kronecker product. Thus, the steering vector $\widetilde{\mathbf{a}}(\theta, \phi)$ of the DCCA is shown as follows:

$$
\widetilde{\mathbf{a}}(\theta, \phi)=\mathbf{G}(\theta, \phi) \mathbf{T}(\theta, \phi)\left(\mathbf{a}(\theta, \phi) \otimes \mathbf{I}_{2}\right)=\left[\widetilde{a}_{\theta}(\theta, \phi), \tilde{a}_{\phi}(\theta, \phi)\right] .
$$

Assume that there are $K$ sources $s_{i}(t), i=1,2, \ldots, K$ in the space. They impinge on the array at certain angles $\left(\theta_{1}, \phi_{1}\right),\left(\theta_{2}, \phi_{2}\right), \ldots,\left(\theta_{K}, \phi_{K}\right)$, where $\theta_{i}$ and $\phi_{i}$ are elevation and azimuth of the $i$ th signal. The equivalent array manifold of the array can be expressed as

$$
\widetilde{\mathbf{A}}=\left[\widetilde{\mathbf{a}}_{\theta}\left(\theta_{1}, \phi_{1}\right), \widetilde{\mathbf{a}}_{\phi}\left(\theta_{1}, \phi_{1}\right), \ldots, \widetilde{\mathbf{a}}_{\theta}\left(\theta_{K}, \phi_{K}\right), \widetilde{\mathbf{a}}_{\phi}\left(\theta_{K}, \phi_{K}\right)\right] .
$$

Each polarized signal can be decomposed into two orthogonal polarization signal components, which can be regarded as two coherent components with the same direction but different polarization.

$$
\left[\begin{array}{c}
s_{i \theta}(t) \\
s_{i \phi}(t)
\end{array}\right]=\left[\begin{array}{c}
\sin \gamma_{i} e^{j \eta_{i}} \\
\cos \gamma_{i}
\end{array}\right] s_{i}(t),
$$

where $\gamma_{i}$ and $\eta_{i}$ represent the auxiliary polarization angle and polarization phase difference of the $i$ th signal. Define the matrix $\mathbf{P}$ containing all polarization parameter information.

$$
\mathbf{P}=\left[\begin{array}{cccc}
\mathbf{p}\left(\gamma_{1}, \eta_{1}\right) & \mathbf{0} & \ldots & \mathbf{0} \\
\mathbf{0} & \mathbf{p}\left(\gamma_{2}, \eta_{2}\right) & \ldots & \mathbf{0} \\
\vdots & \vdots & \ddots & \vdots \\
\mathbf{0} & \mathbf{0} & \ldots & \mathbf{p}\left(\gamma_{K}, \eta_{K}\right)
\end{array}\right]
$$

where $\mathbf{p}\left(\gamma_{i}, \eta_{i}\right)=\left[\sin \gamma_{i} e^{j \eta_{i}}, \cos \gamma_{i}\right]^{\mathrm{T}}$.

Thus, the array output can be rewritten as

$$
\mathbf{x}(t)=\widetilde{\mathbf{A}} \mathbf{P} \mathbf{s}(t)+\mathbf{n}(t)
$$

where

$$
\begin{aligned}
\mathbf{s}(t) & =\left[s_{1}(t), \ldots, s_{K}(t)\right]^{\mathrm{T}} \\
\mathbf{n}(t) & =\left[n_{1 \theta}(t), n_{1 \phi}(t), \ldots, n_{M N \theta}(t), n_{M N \phi}(t)\right]^{\mathrm{T}},
\end{aligned}
$$

$\mathbf{s}(t)$ represents the $K \times 1$ vectors of the incident signals. $\mathbf{n}(t)$ represents the $2 M N \times 1$ zero-mean white Gaussian noise vector with variants $\sigma_{n}^{2}$.

\subsection{Dual-Polarized Cylindrical Conformal Array with Mutual} Coupling. Considering the influence of mutual coupling on the array, the array manifold should be corrected. Assume that each element in the cylindrical array can only receive the electromagnetic coupling interference generated by copolarization component of adjacent elements. $c_{x}, c_{z}$, and $c_{x z}$ are the mutual coupling coefficients between elements, as shown in Figure 2 [15].

According to the spatial structure of the cylindrical array, the mutual coupling matrix of the array can be expressed as follows:

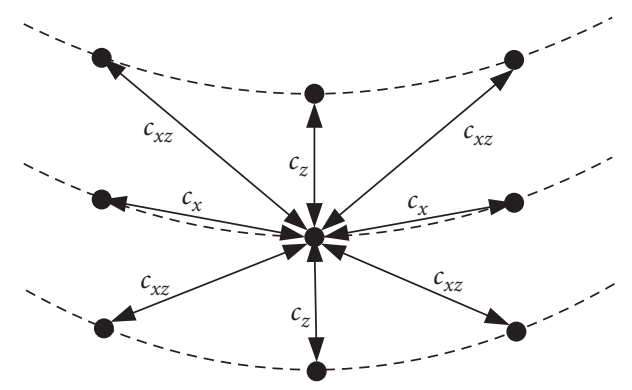

Figure 2: Schematic diagram of mutual coupling of DCCA.

$$
\begin{aligned}
\mathbf{C} & =\left[\begin{array}{ccccc}
\mathbf{C}_{1} & \mathbf{C}_{2} & \mathbf{0} & \cdots & \mathbf{0} \\
\mathbf{C}_{2} & \mathbf{C}_{1} & \mathbf{C}_{2} & \cdots & \mathbf{0} \\
\vdots & \vdots & \ddots & \vdots & \vdots \\
\mathbf{0} & \cdots & \mathbf{C}_{2} & \mathbf{C}_{1} & \mathbf{C}_{2} \\
\mathbf{0} & \cdots & \mathbf{0} & \mathbf{C}_{2} & \mathbf{C}_{1}
\end{array}\right]_{2 M N \times 2 M N} \\
\mathbf{C}_{1} & =\operatorname{Toeplitz}\left\{\left[1,0, c_{x}, 0, \ldots, 0, c_{x}, 0\right]\right\} \\
\mathbf{C}_{2} & =\operatorname{Toeplitz}\left\{\left[c_{z}, 0, c_{x z}, 0, \ldots, 0, c_{x z}, 0\right]\right\} .
\end{aligned}
$$

Considering the effect of mutual coupling, (9) can be rewritten as

$$
\mathbf{x}(t)=\mathbf{C A} \mathbf{A} \mathbf{s}(t)+\mathbf{n}(t) .
$$

Thus, the covariance matrix of the received signal can be expressed as

$$
\mathbf{R}_{x}=E\left\{\mathbf{x}(t) \mathbf{x}^{\mathrm{H}}(t)\right\}=\mathbf{C} \widetilde{\mathbf{A}} \widetilde{\mathbf{R}}_{s} \widetilde{\mathbf{A}}^{\mathrm{H}} \mathbf{C}^{\mathrm{H}}+\sigma_{n}^{2} \mathbf{I}_{x},
$$

where $\widetilde{\mathbf{R}}_{s}=\mathbf{P} \mathbf{R}_{s} \mathbf{P}^{\mathrm{H}}$. $\mathbf{R}_{s}$ is a $K \times K$ matrix, representing the covariance matrix of the signal source. $\sigma_{n}^{2}$ is the noise power and $\mathbf{I}_{x}$ is the $2 M N \times 2 M N$ identity matrix.

\section{Multiparameter Joint Estimation Algorithm}

Simultaneous estimation of multiple parameters in the array brings a large amount of calculation, which is almost impossible to realize. In this section, a stepped multiparameter joint estimation algorithm is proposed based on the dualpolarized cylindrical conformal array in the presence of mutual coupling. The 2D DOA estimation can be divided into two 1D DOA estimations. Elevation can firstly be estimated with the GEESE algorithm proposed in this section. Then, using the estimated elevation result, azimuth and polarization parameters can be estimated through the constructed space spectrum. Finally, the angles of the signals can be reestimated to improve the accuracy of DOA estimation.

3.1. Elevation Estimation. The cylindrical conformal array can be regarded as a linear array composed of multiple uniform circular arrays. Therefore, the dual-polarized cylindrical conformal array has some properties of a uniform linear array, which provides a theoretical basis for proposing the GEESE algorithm. Then, sensors on the boundary of the DCCA are set as auxiliary sensors to eliminate the influence 
of mutual coupling. Afterwards, elevation can be estimated with the GEESE algorithm.

Considering the structural characteristics of the array, the steering vector of the dual-polarized cylindrical conformal array is rewritten as follows:

$$
\widetilde{\mathbf{a}}(\theta, \phi)=\mathbf{G}(\theta, \phi) \mathbf{T}(\theta, \phi)\left\{\left[\mathbf{a}_{z}(\theta) \otimes \mathbf{a}_{x y}(\theta, \phi)\right] \otimes \mathbf{I}_{2}\right\},
$$

where $\mathbf{a}_{z}(\theta)$ is the steering vector of the uniform linear subarray.

$$
\begin{aligned}
& \mathbf{a}_{z}(\theta)=\left[1, \beta_{z}, \beta_{z}^{2}, \ldots, \beta_{z}^{M-1}\right]^{\mathrm{T}} \\
& \beta_{z}(\theta)=\exp \left\{j \frac{2 \pi}{\lambda} d_{z} \cos \theta\right\} .
\end{aligned}
$$

$\mathbf{a}_{x y}(\theta, \phi)$ is the steering vector of the uniform circular subarray.

$$
\begin{aligned}
\mathbf{a}_{x y}(\theta, \phi) & =\left[\beta_{x y 1}, \ldots, \beta_{x y N}\right]^{\mathrm{T}} \\
\beta_{x y j}(\theta, \phi) & =\exp \left\{-j \frac{2 \pi}{\lambda}\left(x_{j} \sin \theta \cos \phi+y_{j} \sin \theta \sin \phi\right)\right\},
\end{aligned}
$$

where $j=1,2, \ldots, N$. According to the characteristics of Kronecker product, (17) is rewritten as

$$
\begin{aligned}
\widetilde{\mathbf{a}}(\theta, \phi) & =\mathbf{G}(\theta, \phi) \mathbf{T}(\theta, \phi)\left[\mathbf{a}_{z}(\theta) \otimes\left(\mathbf{a}_{x y}(\theta, \phi) \otimes \mathbf{I}_{2}\right)\right] \\
& =\mathbf{a}_{z}(\theta) \otimes\left[\mathbf{G}(\theta, \phi) \mathbf{T}(\theta, \phi)\left(\mathbf{a}_{x y}(\theta, \phi) \otimes \mathbf{I}_{2}\right)\right] \\
& =\mathbf{a}_{z}(\theta) \otimes \widetilde{a}_{x y}(\theta, \phi),
\end{aligned}
$$

where $\widetilde{\mathbf{a}}_{x y}(\theta, \phi)=\mathbf{G}(\theta, \phi) \mathbf{T}(\theta, \phi)\left[\mathbf{a}_{x y}(\theta, \phi) \otimes \mathbf{I}_{2}\right]$.

Therefore, the steering vector of the dual-polarized cylindrical conformal array can be defined as the Kronecker product of a uniform linear array and a dual-polarized uniform circular conformal array. Then, considering the existence of mutual coupling, the true steering vector is as follows:

$$
\mathbf{C a}(\theta, \phi)=\mathbf{C}\left(\mathbf{a}_{z}(\theta) \otimes \tilde{\mathbf{a}}_{x y}(\theta, \phi)\right) .
$$

The premise of the GEESE algorithm is that the two subarrays divided by the array have similar displacement vectors in space. In order to meet the above condition, it is necessary to discard the top and bottom layer elements of the dual-polarized cylindrical array. Then, divide two subarrays with similar spatial structures from the remaining elements,

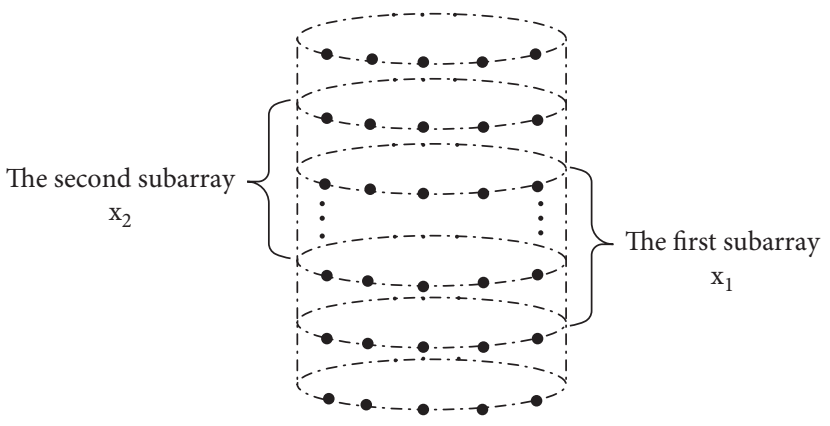

FIgURE 3: Spatial structure of subarray.

as shown in Figure 3. Define two $2(M-3) N \times 2 M N$ selection matrices $\mathbf{P}_{1}$ and $\mathbf{P}_{2}$ to extract the subarrays.where $\mathbf{0}$ represents $2 \mathrm{~N} \times 2 \mathrm{~N}$ zero matrix. I represents the $2 \mathrm{~N} \times 2 \mathrm{~N}$ identity matrix. The output vector of the two subarrays can be expressed by the following equations.

$$
\left\{\begin{array}{l}
\mathbf{x}_{1}(t)=\mathbf{P}_{1} \mathbf{x}(t)=\mathbf{P}_{1} \mathbf{C} \tilde{\mathbf{A}} \mathbf{P}_{s}(t)+\mathbf{P}_{1} \mathbf{n}(t), \\
\mathbf{x}_{2}(t)=\mathbf{P}_{2} \mathbf{x}(t)=\mathbf{P}_{2} \mathbf{C A} \mathbf{P} s(t)+\mathbf{P}_{2} \mathbf{n}(t)
\end{array}\right.
$$

$$
\begin{aligned}
& \mathbf{P}_{1}=\left[\begin{array}{ccccccc}
\mathbf{0} & \mathbf{I} & \mathbf{0} & \cdots & \mathbf{0} & \mathbf{0} & \mathbf{0} \\
\mathbf{0} & \mathbf{0} & \mathbf{I} & \cdots & \mathbf{0} & \mathbf{0} & \mathbf{0} \\
\vdots & \vdots & \vdots & \vdots & \ddots & \vdots & \vdots \\
\mathbf{0} & \mathbf{0} & \mathbf{0} & \cdots & \mathbf{I} & \mathbf{0} & \mathbf{0}
\end{array}\right] \\
& \mathbf{P}_{2}=\left[\begin{array}{ccccccc}
\mathbf{0} & \mathbf{0} & \mathbf{I} & \mathbf{0} & \cdots & \mathbf{0} & \mathbf{0} \\
\mathbf{0} & \mathbf{0} & \mathbf{0} & \mathbf{I} & \cdots & \mathbf{0} & \mathbf{0} \\
\vdots & \vdots & \vdots & \vdots & \vdots & \vdots & \vdots \\
\mathbf{0} & \mathbf{0} & \mathbf{0} & \cdots & \mathbf{0} & \mathbf{I} & \mathbf{0}
\end{array}\right],
\end{aligned}
$$

Thus, the true steering vectors of the two subarrays can be expressed as $\mathbf{P}_{1} \mathbf{C} \tilde{\mathbf{a}}(\theta, \phi)$ and $\mathbf{P}_{2} \mathbf{C} \tilde{\mathbf{a}}(\theta, \phi)$. The steering vectors in the $\theta$ polarization and $\phi$ polarization directions, respectively (22), can be expressed as follows:

$$
\begin{aligned}
\tilde{a}(\theta, \phi) & =a_{z}(\theta) \otimes\left[\tilde{a}_{x y \theta}(\theta, \phi), \tilde{a}_{x y \phi}(\theta, \phi)\right] \\
& =\left[\tilde{a}_{\theta}(\theta, \phi), \tilde{a}_{\phi}(\theta, \phi)\right],
\end{aligned}
$$

where $\tilde{a}_{x y \theta}(\theta, \phi)$ and $\tilde{a}_{x y \phi}(\theta, \phi)$ are $2 N \times 1$ steering vectors and $\tilde{a}_{\theta}(\theta, \phi)$ and $\tilde{a}_{\phi}(\theta, \phi)$ are $2 M N \times 1$ steering vector matrices. Taking the $\theta$ polarization direction as an example, the true steering vectors of the two subarrays $P_{1} C \widetilde{a}_{\theta}$ and $P_{2} C \tilde{a}_{\theta}$ are as follows: 


$$
\begin{aligned}
& P_{1} C \widetilde{a}_{\theta}=\left[\begin{array}{ccccccc}
0 & I & 0 & \cdots & 0 & 0 & 0 \\
0 & 0 & I & \cdots & 0 & 0 & 0 \\
\vdots & \vdots & \vdots & \cdots & \vdots & \vdots & \vdots \\
0 & 0 & 0 & \cdots & I & 0 & 0
\end{array}\right]\left[\begin{array}{ccccc}
\mathbf{C}_{1} & \mathbf{C}_{2} & \mathbf{0} & \cdots & \mathbf{0} \\
\mathbf{C}_{2} & \mathbf{C}_{1} & \mathbf{C}_{2} & \cdots & 0 \\
\vdots & \vdots & \ddots & \vdots & \vdots \\
\mathbf{0} & \cdots & \mathbf{C}_{2} & \mathbf{C}_{1} & \mathbf{C}_{2} \\
\mathbf{0} & \cdots & \mathbf{0} & \mathbf{C}_{2} & \mathbf{C}_{1}
\end{array}\right] . \\
& {\left[\begin{array}{c}
\widetilde{\mathbf{a}}_{x y \theta} \\
\beta_{z} \widetilde{\mathbf{a}}_{x y \theta} \\
\vdots \\
\beta_{z}^{M-1} \widetilde{\mathbf{a}}_{x y \theta}
\end{array}\right]} \\
& =\left[\begin{array}{c}
\mathbf{C}_{2} \widetilde{\mathbf{a}}_{x y \theta}+\mathbf{C}_{1} \beta_{z} \widetilde{\mathbf{a}}_{x y \theta}+\mathbf{C}_{2} \beta_{z}^{2} \widetilde{\mathbf{a}}_{x y \theta} \\
\mathbf{C}_{2} \beta_{z} \widetilde{\mathbf{a}}_{x y \theta}+\mathbf{C}_{1} \beta_{z}^{2} \widetilde{\mathbf{a}}_{x y \theta}+\mathbf{C}_{2} \beta_{z}^{3} \widetilde{\mathbf{a}}_{x y \theta} \\
\vdots \\
\mathbf{C}_{2} \beta_{z}^{M-4} \widetilde{\mathbf{a}}_{x y \theta}+\mathbf{C}_{1} \beta_{z}^{M-3} \widetilde{\mathbf{a}}_{x y \theta}+\mathbf{C}_{2} \beta_{z}^{M-2} \widetilde{\mathbf{a}}_{x y \theta}
\end{array}\right]
\end{aligned}
$$

$$
\begin{aligned}
& P_{1} C \tilde{a}_{\theta}=\left[\begin{array}{ccccccc}
0 & I & 0 & \cdots & 0 & 0 & 0 \\
0 & 0 & I & \cdots & 0 & 0 & 0 \\
\vdots & \vdots & \vdots & \cdots & \vdots & \vdots & \vdots \\
0 & 0 & 0 & \cdots & I & 0 & 0
\end{array}\right]\left[\begin{array}{ccccc}
\mathrm{C}_{1} & \mathbf{C}_{2} & \mathbf{0} & \cdots & \mathbf{0} \\
\mathbf{C}_{2} & \mathbf{C}_{1} & \mathbf{C}_{2} & \cdots & \mathbf{0} \\
\vdots & \vdots & \ddots & \vdots & \vdots \\
\mathbf{0} & \cdots & \mathbf{C}_{2} & \mathbf{C}_{1} & \mathbf{C}_{2} \\
\mathbf{0} & \cdots & \mathbf{0} & \mathbf{C}_{2} & \mathbf{C}_{1}
\end{array}\right] . \\
& {\left[\begin{array}{c}
\widetilde{\mathbf{a}}_{x y \theta} \\
\beta_{z} \widetilde{\mathbf{a}}_{x y \theta} \\
\vdots \\
\beta_{z}^{M-1} \widetilde{\mathbf{a}}_{x y \theta}
\end{array}\right]} \\
& =\left[\begin{array}{c}
\mathbf{C}_{2} \widetilde{\mathbf{a}}_{x y \theta}+\mathbf{C}_{1} \beta_{z} \widetilde{\mathbf{a}}_{x y \theta}+\mathbf{C}_{2} \beta_{z}^{2} \widetilde{\mathbf{a}}_{x y \theta} \\
\mathbf{C}_{2} \beta_{z} \widetilde{\mathbf{a}}_{x y \theta}+\mathbf{C}_{1} \beta_{z}^{2} \widetilde{\mathbf{a}}_{x y \theta}+\mathbf{C}_{2} \beta_{z}^{3} \widetilde{\mathbf{a}}_{x y \theta} \\
\vdots \\
\mathbf{C}_{2} \beta_{z}^{M-4} \widetilde{\mathbf{a}}_{x y \theta}+\mathbf{C}_{1} \beta_{z}^{M-3} \widetilde{\mathbf{a}}_{x y \theta}+\mathbf{C}_{2} \beta_{z}^{M-2} \widetilde{\mathbf{a}}_{x y \theta}
\end{array}\right]
\end{aligned}
$$

From (28)-(29), the following equation can be obtained:

$$
\mathbf{P}_{2} \mathbf{C} \tilde{\mathbf{a}}_{\theta}=\mathbf{P}_{1} \mathbf{C} \tilde{\mathbf{a}}_{\theta} \beta_{z} .
$$

The $\phi$ polarization component has similar results, and the following equation can be obtained:

$$
\mathbf{P}_{2} \mathbf{C} \widetilde{\mathbf{a}}_{\phi}=\mathbf{P}_{1} \mathbf{C} \widetilde{\mathbf{a}}_{\phi} \beta_{z}
$$

when there are $K$ sources in the space. Moreover, the following equation can be obtained:

$$
\begin{aligned}
\mathbf{P}_{2} C \tilde{A} P & =\mathbf{P}_{1} C \tilde{A}\left(\boldsymbol{\Phi} \otimes \mathbf{I}_{2}\right) \mathbf{P} \\
& =\mathbf{P}_{1} C \tilde{A} P \boldsymbol{\Phi},
\end{aligned}
$$

where $\Phi=\operatorname{diag}\left\{\beta_{z 1}, \beta_{z 2}, \ldots, \beta_{z K}\right\}$ is a $K \times K$ matrix containing the elevation information but not mixed with other information. $\beta_{z i}$ is the simplified representation of $\beta_{z}\left(\theta_{i}\right) . \mathbf{a}_{\theta}$ and $\widetilde{\mathbf{a}}_{x y \theta}$ are simplified representations of $\widetilde{\mathbf{a}}_{\theta}(\theta, \phi)$ and $\widetilde{\mathbf{a}}_{x y \theta}(\theta, \phi)$, respectively. Thus, the following equation can be obtained:

$$
\left\{\begin{array}{l}
\mathbf{x}_{1}(t)=\mathbf{P}_{1} \mathbf{x}(t)=\mathbf{P}_{1} \mathbf{C} \tilde{\mathbf{A}} \mathbf{P} \mathbf{s}(t)+\mathbf{P}_{1} \mathbf{n}(t), \\
\mathbf{x}_{2}(t)=\mathbf{P}_{2} \mathbf{x}(t)=\mathbf{P}_{1} \mathbf{C A} P \boldsymbol{A} s(t)+\mathbf{P}_{2} \mathbf{n}(t) .
\end{array}\right.
$$

Then, the GEESE algorithm can be used to estimate the elevations. The signal subspace $U_{s}$ can be obtained through the eigenvalue decomposition of the covariance matrix $\mathbf{R}_{x}$. Define $\mathbf{U}_{s 1}=\mathbf{P}_{1} \mathbf{U}_{s}, \mathbf{U}_{s 2}=\mathbf{P}_{2} \mathbf{U}_{s}$. The signal subspace spanned by the eigenvectors corresponding to the eigenvalues is the same as the subspace spanned by the array manifold matrix [16]. Thus, there is a $K \times K$ full rank matrix $\mathbf{T}$ that satisfied the following equation:

$$
\left[\begin{array}{l}
\mathbf{U}_{s 1} \\
\mathbf{U}_{s 2}
\end{array}\right]=\left[\begin{array}{c}
\overline{\mathbf{A}} \mathbf{T} \\
\overline{\mathbf{A}} \boldsymbol{\Phi} \mathbf{T}
\end{array}\right]
$$

where $\overline{\mathbf{A}}=\mathbf{P}_{1} \mathbf{C A} P . v_{i}=\left\{v_{1}, v_{2}, \ldots, v_{K}\right\}$ is defined as the generalized eigenvalue of the matrix pair $\left\{\mathbf{U}_{s 1}^{\mathrm{H}} \mathbf{U}_{s 1}, \mathbf{U}_{s 1}^{\mathrm{H}} \mathbf{U}_{s 2}\right\}$.

$$
\begin{aligned}
\left|\mathbf{U}_{s 1}^{\mathrm{H}} \mathbf{U}_{s 1}-\boldsymbol{v}_{i} \mathbf{U}_{s 1}^{\mathrm{H}} \mathbf{U}_{s 2}\right| & =\left|\mathbf{T}^{\mathrm{H}} \bar{A}^{\mathrm{H}} \overline{\mathbf{A}}\left(\mathbf{I}_{K}-\boldsymbol{v}_{i} \boldsymbol{\Phi}\right) \mathbf{T}\right| \\
& =\mathbf{0}(i=1,2, \ldots, K) .
\end{aligned}
$$

Since the matrix $\mathbf{T}$ and array manifold $\overline{\mathbf{A}}$ have the same rank $K$, it can be obtained from formula (26):

$$
\left|\mathbf{I}_{K}-\boldsymbol{v}_{i} \boldsymbol{\Phi}\right|=\mathbf{0} \text {. }
$$

Thus, the eigenvalues of matrix $\Phi$ are equal to $v_{i}$. The elevation estimator can be estimated.

$$
\theta_{i}=\cos ^{-1}\left(\frac{\lambda}{2 \pi d_{z}} \arg \left(v_{i}\right)\right)
$$

where "arg $(x)$ " represents the phase of $x$.

3.2. Azimuth and Polarization Parameter Estimation. Each polarized signal is regarded as two orthogonal polarized components. The two components can be treated as a pair of coherent signals that have the same direction but different polarization. The traditional decoherence methods are only suitable for planar arrays but not suitable for conformal arrays. Therefore, the dual-polarized cylindrical conformal array must be projected into a rectangular array. After using the projection transformation and spatial smoothing algorithm, the dual-polarized cylindrical conformal array signal can make decoherence.

The projection operator satisfies $\mathbf{P}_{\mathrm{A} \mid \mathrm{U}} \mathbf{C} \widetilde{\mathbf{A}}=\mathbf{A}_{v}$ and $\mathbf{P}_{\mathrm{A} \mid \mathrm{U}} \mathbf{U}_{n}=\mathbf{0}$ [17]. $\mathbf{A}_{v}$ is the array manifold of the virtualized dual-polarized rectangular array, which will be used as the signal subspace of the oblique projection. $\mathbf{U}_{n}$ is the noise subspace after eigendecomposition of the covariance matrix of equation (16). The orthogonal projection matrix $\mathbf{P}_{U}^{\perp}$ of the noise subspace $\mathbf{U}_{n}$ is shown as follows:

$$
\begin{aligned}
& \mathbf{P}_{\mathrm{U}}^{\perp}=\mathbf{I}_{\mathrm{U}}-\mathbf{P}_{\mathrm{U}}, \\
& \mathbf{P}_{\mathrm{U}}=\mathbf{U}_{n}\left(\mathbf{U}_{n}^{\mathrm{H}} \mathbf{U}_{n}\right)^{-1} \mathbf{U}_{n}^{\mathrm{H}},
\end{aligned}
$$

where $\mathbf{I}_{U}$ is a $2 M N \times 2 M N$ identity matrix.

Then, the oblique projection matrix $\mathbf{P}_{\mathrm{A} \mid \mathrm{U}}$ is expressed as follows: 


$$
\mathbf{P}_{\mathrm{A} \mid \mathrm{U}}=\mathbf{A}_{v}\left[(\mathbf{C} \tilde{\mathbf{A}})^{\mathrm{H}} \mathbf{P}_{\mathrm{U}}^{\perp}(\mathbf{C} \tilde{\mathbf{A}})\right]^{-1}(\mathbf{C} \tilde{\mathbf{A}})^{\mathrm{H}} \mathbf{P}_{\mathrm{U}}^{\perp}
$$

From (16), the projected covariance matrix is written as follows:

$$
\begin{aligned}
\mathbf{R} & =\mathbf{P}_{\mathrm{A} \mid \mathrm{U}}^{\mathrm{H}} \mathbf{R}_{x} \mathbf{P}_{\mathrm{A} \mid \mathrm{U}} \\
& =\mathbf{P}_{\mathrm{A} \mid \mathrm{U}}^{\mathrm{H}} \mathbf{C} \widetilde{\mathbf{A}} \widetilde{R}_{s}\left(\mathbf{P}_{\mathrm{A} \mid \mathrm{U}}^{\mathrm{H}} \mathbf{C} \widetilde{\mathbf{A}}\right)^{\mathrm{H}}+\sigma_{n}^{2} \mathbf{P}_{\mathrm{A} \mid \mathrm{U}}^{\mathrm{H}} \mathbf{P}_{\mathrm{A} \mid \mathrm{U}} \cdot
\end{aligned}
$$

For the existence of the projection matrix, the noise will no longer be white. In order to reconstruct white noise, the new projection matrix is defined as follows:

$$
\widetilde{P}_{\mathrm{A} \mid \mathrm{U}}=\left(\mathbf{P}_{\mathrm{A} \mid \mathrm{U}}^{\mathrm{H}} \mathbf{P}_{\mathrm{A} \mid \mathrm{U}}\right)^{-1 / 2} \mathbf{P}_{\mathrm{A} \mid \mathrm{U}}^{\mathrm{H}} .
$$

Thus, the projected covariance matrix is rewritten as follows:

$$
\widetilde{\mathbf{R}}=\widetilde{P}_{\mathrm{A} \mid \mathrm{U}} \mathbf{R}_{x} \widetilde{P}_{\mathrm{A} \mid \mathrm{U}}^{\mathrm{H}}
$$

Due to the particularity of a dual-polarized conformal array, smoothing should be carried out between copolarization components. Through the following forward and backward smoothing, a new covariance matrix can be constructed as follows [18]:

$$
\begin{aligned}
\mathbf{R}^{f b} & =\frac{\widetilde{\mathbf{R}}+\mathbf{D} \widetilde{\mathbf{R}}^{*} \mathbf{D}}{2} \\
\mathbf{D} & =\left[\begin{array}{llllll}
\mathbf{J}_{\mathrm{D}} & \mathbf{0} & \mathbf{0} & \mathbf{0} & \mathbf{0} \\
\mathbf{0} & \mathbf{J}_{\mathrm{D}} & \mathbf{0} & \mathbf{0} & \mathbf{0} \\
\mathbf{0} & \mathbf{0} & \ddots & \mathbf{0} & \mathbf{0} \\
\mathbf{0} & \mathbf{0} & \mathbf{0} & \mathbf{J}_{\mathrm{D}} & \mathbf{0} \\
\mathbf{0} & \mathbf{0} & \mathbf{0} & \mathbf{0} & \mathbf{J}_{\mathrm{D}}
\end{array}\right],
\end{aligned}
$$

where $\mathbf{D}=\mathbf{I}_{M} \otimes \mathbf{J}_{\mathrm{D}}$ is the dual-polarized forward and backward smoothing matrix. Matrix $D$ ensures that the copolarized components are smoothed, and the cross-polarized components do not affect each other. $\mathbf{J}_{\mathrm{D}}=\mathbf{J}_{N} \otimes \mathbf{I}_{2}$ is a $2 N \times 2 N$ matrix. $\mathbf{I}_{2}$ is a $2 \times 2$ identity matrix, $\mathbf{J}_{N}$ is a $N \times N$ inverse identity diagonal matrix, and $\mathbf{I}_{M}$ is a $M \times M$ identity matrix. " $(\cdot)^{*}$ " represents complex conjugate of the matrix. The proof process is shown in Appendix A.

Through the above smoothing, the rank of covariance matrix is restored from $K$ to $2 K$, and then $\mathbf{R}^{f b}$ is expressed as follows:

$$
\mathbf{R}^{f b}=\mathbf{U}_{s}^{f b} \boldsymbol{\Lambda}_{s} \mathbf{U}_{s}^{f b}+\mathbf{U}_{n}^{f b} \boldsymbol{\Lambda}_{n} \mathbf{U}_{n}^{f b}
$$

$\mathbf{U}_{s}^{f b}$ is a $2 M N \times 2 K$ matrix and $\mathbf{U}_{n}^{f b}$ is a $2 M N \times(2 M N-2 K)$ matrix. $\Lambda_{s}$ and $\Lambda_{n}$ are the diagonal matrices composed of corresponding eigenvalues.

According to the principle of subspace orthogonality, the steering vector of the decoherent array signal is orthogonal to the noise subspace. The following equation is satisfied:

$$
\mathbf{U}_{n}^{f b \mathrm{H}} \widetilde{\mathbf{P}}_{\mathrm{A} \mid \mathrm{U}} \mathbf{C} \widetilde{a}\left(\theta_{i}, \phi_{i}\right)=\mathbf{0} \quad(i=1,2, \ldots, K) .
$$

Since we have already got the estimated values of elevation in Subsection 3.1, we can define $Q_{\theta}\left(\widehat{\theta}_{i}, \phi\right)$ and $Q_{\phi}\left(\widehat{\theta}_{i}, \phi\right)$ to construct the spatial estimation spectrum:

$$
\left\{\begin{array}{l}
\mathrm{Q}_{\theta}\left(\widehat{\theta}_{i}, \phi\right)=\left\|\mathbf{U}_{n}^{f b \mathrm{H}} \widetilde{\mathbf{P}}_{\mathrm{A} \mid \mathrm{U}} \mathbf{C} \widetilde{a}_{\theta}\left(\widehat{\theta}_{i}, \phi\right)\right\|^{2}, \\
\mathrm{Q}_{\phi}\left(\widehat{\theta}_{i}, \phi\right)=\left\|\mathbf{U}_{n}^{f b \mathrm{H}} \widetilde{\mathbf{P}}_{\mathrm{A} \mid \mathrm{U}} \mathbf{C} \widetilde{a}_{\phi}\left(\widehat{\theta}_{i}, \phi\right)\right\|^{2} .
\end{array}\right.
$$

When the scanning angle is equal to the actual arrival angle of the signal, at least one of the above two functions is zero. Therefore, the following spectrum can be constructed to estimate the azimuth:

$$
P\left(\widehat{\theta}_{i}, \phi\right)=\frac{1}{\min \left[Q_{\theta}\left(\widehat{\theta}_{i}, \phi\right), Q_{\phi}\left(\widehat{\theta}_{i}, \phi\right)\right]} .
$$

The azimuth can be estimated by searching the highest peak of the spatial spectrum.

Consequently, the polarization parameters $\gamma$ and $\eta$ can be estimated using the obtained DOA estimation result $\left(\widehat{\theta}_{i}, \widehat{\phi}_{i}\right)$. The steering vector of the array considering the polarization sensitivity is expressed as follows:

$$
\widetilde{\mathbf{a}}\left(\widehat{\theta}_{i}, \widehat{\phi}_{i}, \gamma, \eta\right)=\widetilde{\mathbf{a}}\left(\hat{\theta}_{i}, \widehat{\phi}_{i}\right) \mathbf{p}(\gamma, \eta) \text {. }
$$

The polarization parameters can be estimated through the following equation:

$$
\left(\widehat{\gamma}_{i}, \widehat{\eta}_{i}\right)=\underset{(\gamma, \eta)}{\arg \max } \frac{1}{\left\|\mathbf{U}_{n}^{\mathrm{H}} \mathbf{C} \widetilde{\mathbf{a}}\left(\widehat{\theta}_{i}, \widehat{\phi}_{i}, \gamma, \eta\right)\right\|^{2}},
$$

where $\mathbf{U}_{n}$ is the noise subspace of $\mathbf{R}_{x}$. Each set of polarization parameters corresponds to the angle estimation.

Using the estimated polarization parameters $\left(\widehat{\gamma}_{i}, \widehat{\eta}_{i}\right)$, the angles of the signals can be reestimated to improve the accuracy of DOA estimation. The steering vector is rewritten as follows:

$$
\widetilde{\mathbf{a}}\left(\theta, \phi, \widehat{\gamma}_{i}, \widehat{\eta}_{i}\right)=\widetilde{\mathbf{a}}(\theta, \phi) \mathbf{p}\left(\widehat{\gamma}_{i}, \widehat{\eta}_{i}\right) .
$$

Then, a spatial spectrum can be constructed according to the orthogonality of the subspace to complete the DOA reestimation:

$$
P(\theta, \phi)=\sum_{i=1}^{K} \frac{1}{\left\|\mathbf{U}_{n}^{\mathrm{H}} \mathbf{C} \widetilde{\mathbf{a}}\left(\theta, \phi, \widehat{\gamma}_{i}, \widehat{\eta}_{i}\right)\right\|^{2}} .
$$

From (42), the reestimation of DOA can be achieved.

\section{Simulation Results}

In the simulation experiment, a dual-polarized cylindrical conformal array with $10 \times 15$ elements is considered. The array elements adopt the dual-polarized patch antennas. The spacing between the adjacent elements is half the wavelength. The patterns of the antenna elements are as follows [19]: 


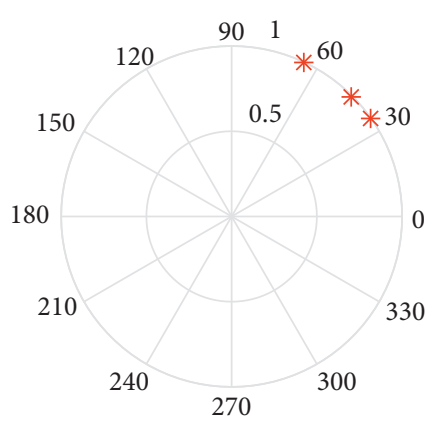

(a)

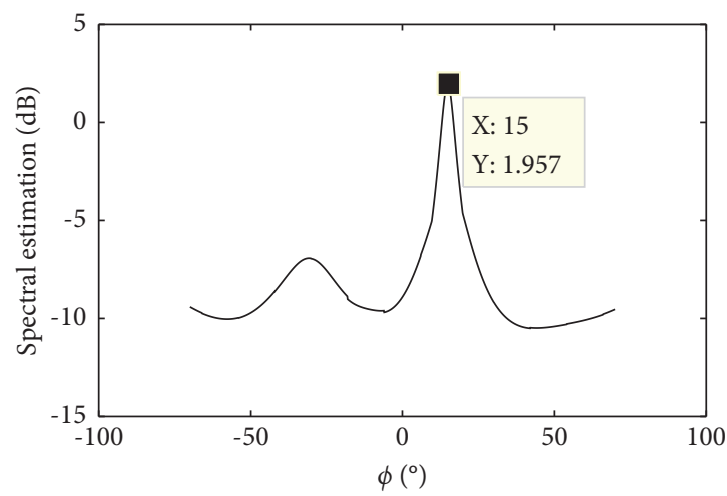

(c)

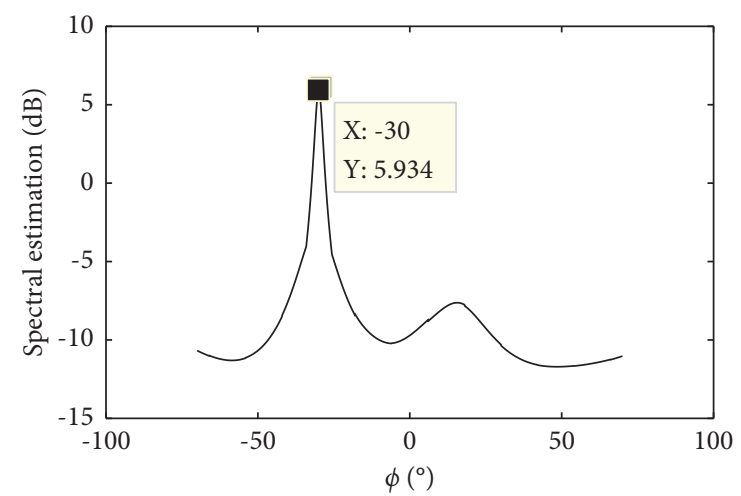

(b)

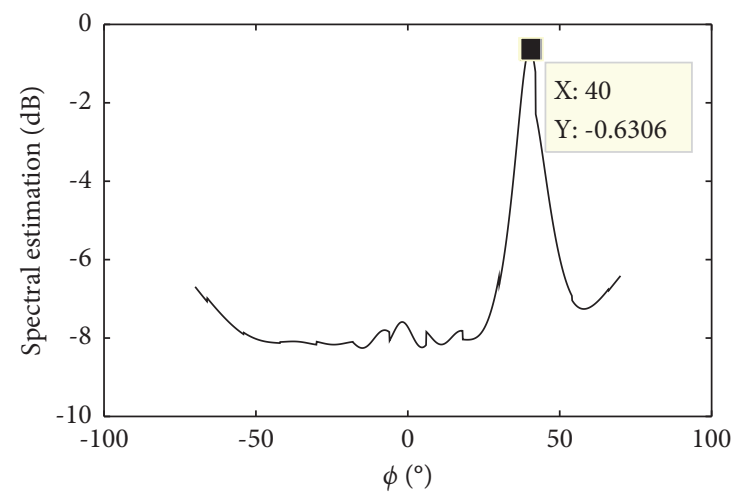

(d)

Figure 4: The DOA initial estimation.

$$
\left\{\begin{array}{l}
g_{\theta}\left(\theta^{\prime}, \phi^{\prime}\right)=\left[J_{2}\left(\frac{\pi d \sin \theta^{\prime}}{\lambda}\right)-J_{0}\left(\frac{\pi d \sin \theta^{\prime}}{\lambda}\right)\right], \\
\cdot\left[\cos \phi^{\prime}-j \sin \phi^{\prime}\right], \frac{0 \leq \theta^{\prime} \leq \pi}{2}, \\
g_{\phi}\left(\theta^{\prime}, \phi^{\prime}\right)=\left[J_{2}\left(\frac{\pi d \sin \theta^{\prime}}{\lambda}\right)+J_{0}\left(\frac{\pi d \sin \theta^{\prime}}{\lambda}\right)\right], \\
\cdot \cos \theta^{\prime}\left[\sin \phi^{\prime}-j \cos \phi^{\prime}\right], \frac{0 \leq \theta^{\prime} \leq \pi}{2}, \\
g_{\theta}\left(\theta^{\prime}, \phi^{\prime}\right)=g_{\phi}\left(\theta^{\prime}, \phi^{\prime}\right)=0, \frac{\theta^{\prime}>\pi}{2},
\end{array}\right.
$$

where $J_{0}$ and $J_{2}$ are the zero-order and second-order Bessel functions of the first kind, respectively.

In the first simulation, we assume there are three independent far-field sources from directions $\left(35^{\circ},-30^{\circ}\right)\left(45^{\circ}, 15^{\circ}\right)\left(65^{\circ}, 40^{\circ}\right)$ in the space impinging on the array. The polarization parameters of the three sources are $\left(20^{\circ}, 60^{\circ}\right)\left(30^{\circ}, 45^{\circ}\right)\left(60^{\circ}, 30^{\circ}\right)$, respectively. We set the scan ranges of the elevation and azimuth to $\left(0^{\circ}, 140^{\circ}\right)$ and $\left(-70^{\circ}, 70^{\circ}\right)$, respectively. And the scan step size is set to $0.1^{\circ}$.
The mutual coupling coefficients are set to $c_{x}=0.5+0.2 \mathrm{i}$, $c_{z}=0.4+0.1 \mathrm{i}$, and $c_{x z}=0.2-0.2 \mathrm{i}$, respectively. The signalto-noise (SNR) is fixed to $10 \mathrm{~dB}$. And the number of the snapshots is fixed to 200 .

The elevation initial estimation result is shown in Figure 4(a). And the spectra of the three azimuth initial estimation are shown in Figures 4(b)-4(d), respectively. Figures 5-7 plot the spectral peaks corresponding to the polarization parameter estimation results. Then, the spatial spectrum obtained by (42) is shown in Figure 8 . From the figure, it can be seen that the spectrum peaks coincide with the real signal DOAs. In order to reduce the amount of calculation, we set the scan step size to ${ }^{2^{\circ}}$ during the angle refined estimation, so there will be an angle estimation deviation of about $1^{\circ}$. Secondary refine scanning can be carried out subsequently with a step of $0.1^{\circ}$ to acquire higher accuracy results. From the simulation results, we can see the proposed multiparameter joint estimation algorithm can realize the estimation of the elevation, azimuth, and polarization parameters, which demonstrate the effectiveness of the proposed algorithm.

Furthermore, Monte Carlo experiments are carried to verify the estimation accuracy of the proposed algorithm. The root mean square error (RMSE) of the parameter estimation is calculated through 100 Monte Carlo experiments $\left(M_{c}=100\right)$ and compared with the CRB lower bound. The derivation of $\mathrm{CRB}$ can refer to in Appendix B. 


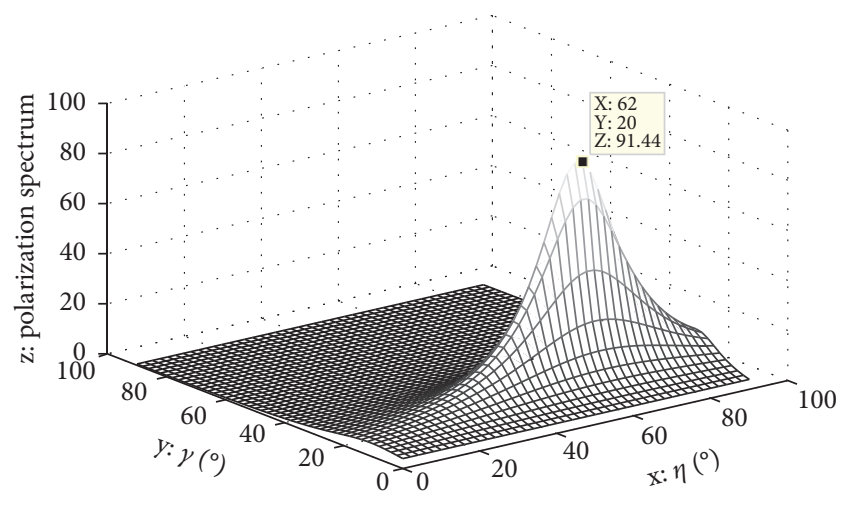

Figure 5: Polarization estimation with $(\theta, \phi)=\left(30^{\circ},-40^{\circ}\right)$.

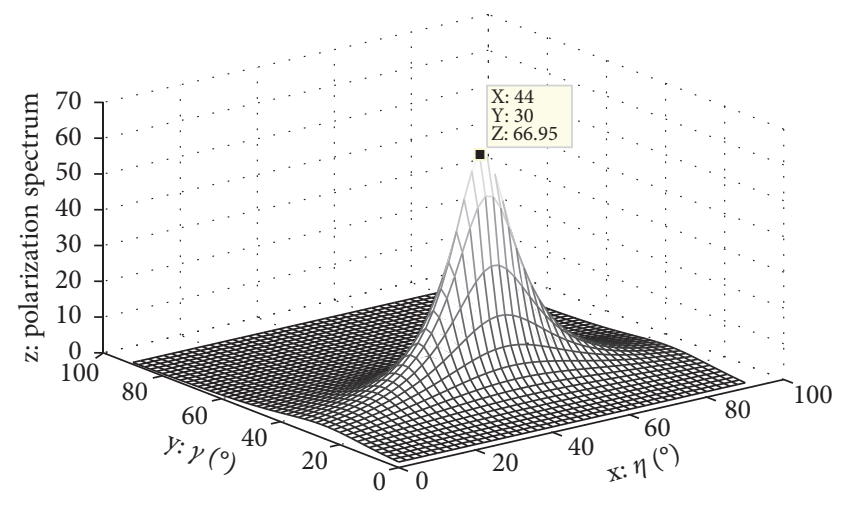

Figure 6: Polarization estimation with $(\theta, \phi)=\left(45^{\circ}, 15^{\circ}\right)$.

The RMSE of DOA and polarization parameters can be defined as follows:

$$
\begin{aligned}
& \operatorname{RMSE}_{\theta}=\frac{1}{\sqrt{M_{c} K}}\left[\sum_{m=1}^{M_{c}}\left[\sum_{i=1}^{K}\left(\theta_{i}-\widehat{\theta}_{i, m}\right)^{2}\right]\right]^{\frac{1}{2}} \\
& \operatorname{RMSE}_{\phi}=\frac{1}{\sqrt{M_{c} K}}\left[\sum_{m=1}^{M_{c}}\left[\sum_{i=1}^{K}\left(\phi_{i}-\widehat{\phi}_{i, m}\right)^{2}\right]\right]^{\frac{1}{2}} \\
& \operatorname{RMSE}_{\gamma}=\frac{1}{\sqrt{M_{c} K}}\left[\sum_{m=1}^{M_{c}}\left[\sum_{i=1}^{K}\left(\gamma_{i}-\widehat{\gamma}_{i, m}\right)^{2}\right]\right]^{\frac{1}{2}} \\
& \operatorname{RMSE}_{\eta}=\frac{1}{\sqrt{M_{c} K}}\left[\sum_{m=1}^{M_{c}}\left[\sum_{i=1}^{K}\left(\eta_{i}-\widehat{\eta}_{i, m}\right)^{2}\right]\right]^{\frac{1}{2}} .
\end{aligned}
$$

In the second simulation, the SNR changes from $0 \mathrm{~dB}$ to $20 \mathrm{~dB}$ with the step of $2 \mathrm{~dB}$. The other conditions are consistent with the first simulation. Figure 9 plots the RMSE curves of initial and refined angle estimation with the SNR increases. And the performance of refined angle estimation is close to the CRB. Figure 10 plots the RMSE curves of polarization parameters for SNR from $0 \mathrm{~dB}$ to $20 \mathrm{~dB}$ with the step of $2 \mathrm{~dB}$.

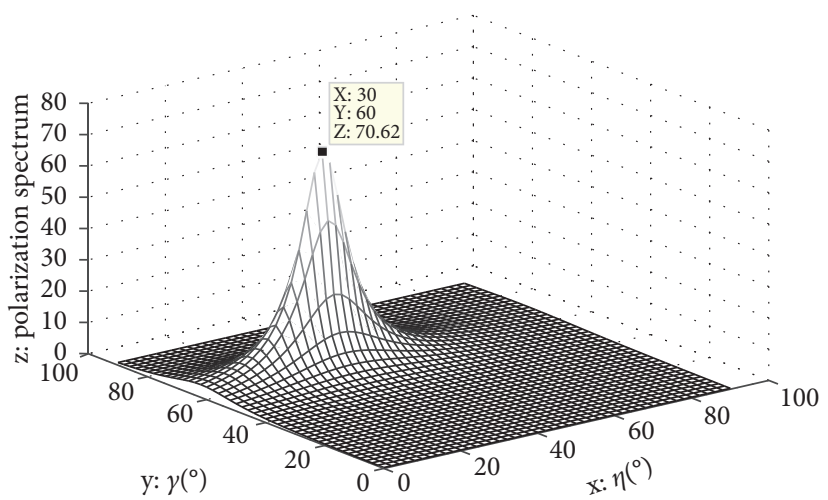

FIgURe 7: Polarization estimation with $(\theta, \phi)=\left(65^{\circ}, 40^{\circ}\right)$.

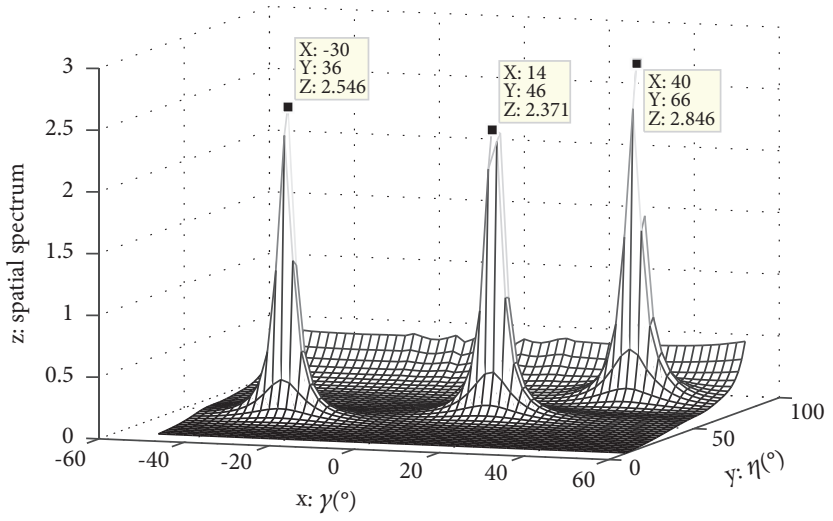

Figure 8: DOA refined estimation result.

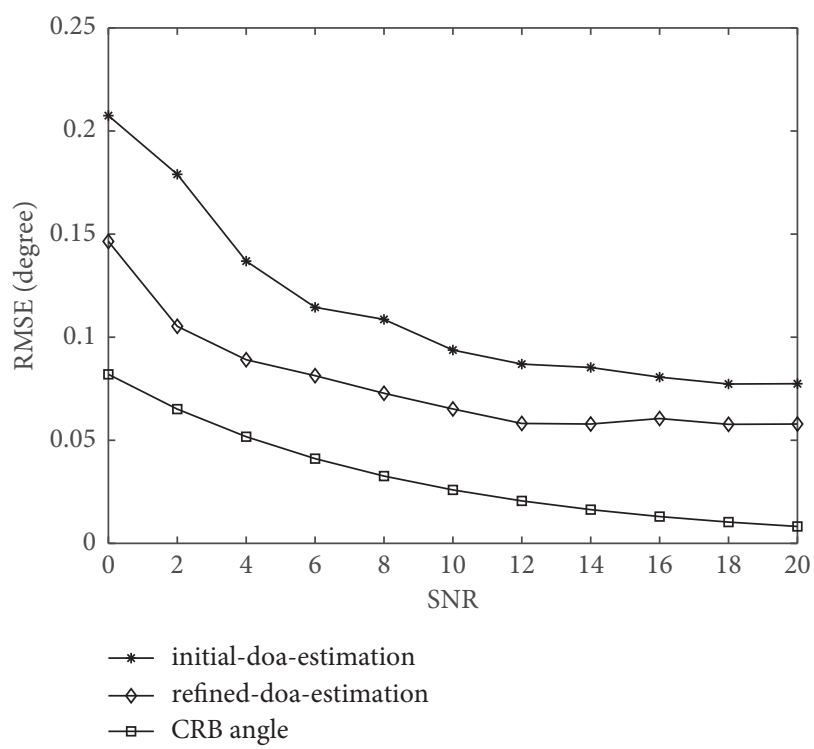

FIGURE 9: RMSE of DOA with different SNR.

In the third simulation, the snapshots change from 100 to 1000 with a step of 100 . And the SNR is fixed to $5 \mathrm{~dB}$. The other conditions are consistent with the first simulation. Figure 11 plots the RMSE curves of initial and refined angle estimation with the snapshots increases. Figure 12 plots the 


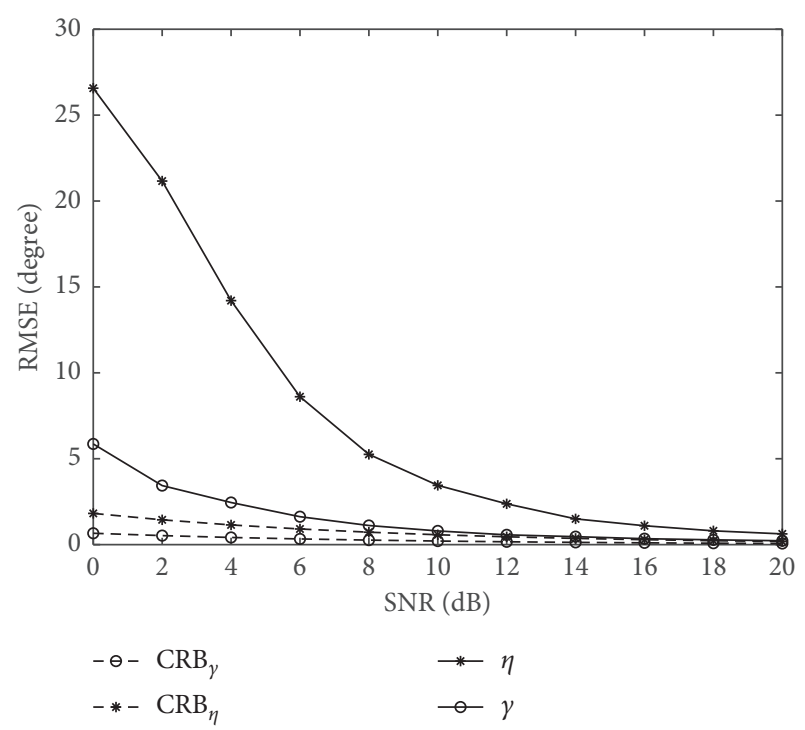

FIGURE 10: RMSE of polarization parameters with different SNR.

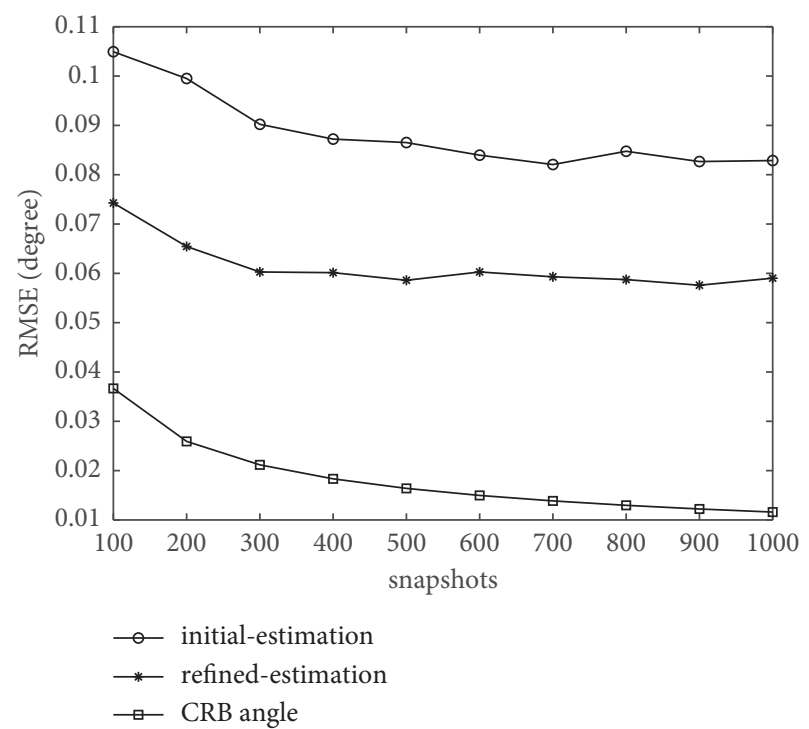

FIgURE 11: RMSE of DOA with different snapshots.

RMSE curves of polarization parameters for snapshots from 100 to 1000 with the step of 100 .

In Figures 9 and 11, the DOA estimation performance gets better and better with the increase of SNR or snapshots. Then, the RMSE curves have a downward trend. With the increase of SNR and snapshots, the DOA estimation RMSE quickly decreases and approaches the Cramer-Rao lower bound.

In Figures 10 and 12, the RMSE values of the polarization parameters estimation gradually decrease regardless of the increase in the SNR or the snapshots. Compared with DOA estimation, the accuracy of polarization parameter estimation is relatively low because the polarization parameter estimation only has two receiving data in the polarization parameter estimation. But in the spatial domain, there are $10 \times 15$ elements for DOA estimation, so it is reasonable that the performance of DOA estimation is higher than that of

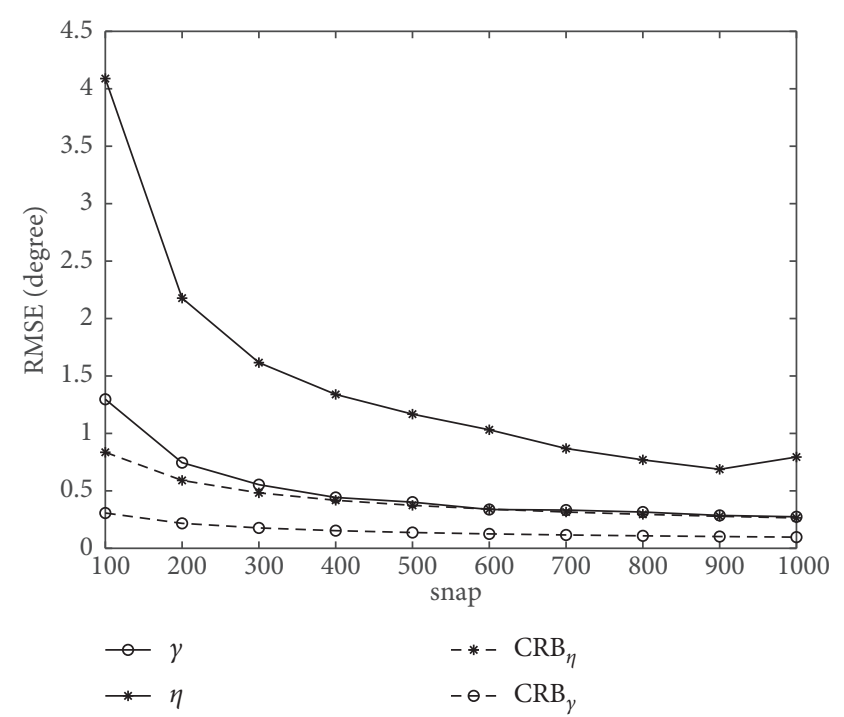

FIGURE 12: RMSE of polarization parameters with different snapshots.

polarization parameter estimation. The RMSE curves of the polarization parameters approach the CRB lower bound curve with the increase of the number of snapshots or SNR, which indicates that the polarization parameter estimation also has good performance.

\section{Conclusion}

A multiparameter joint estimation algorithm has been proposed based on the dual-polarized cylindrical conformal array in the presence of mutual coupling. The 2D DOA estimation is firstly divided into the $1 \mathrm{D}$ estimation of elevation and azimuth. By setting the elements on the boundary of the DCCA as auxiliary sensors, the effect of mutual coupling is proved to be eliminated, and elevation can firstly be estimated by the GEESE algorithm. Then, polarization sensitivity is eliminated by oblique projection transformation and the dual-polarized forward-backward smoothing technique. After eliminating the influence of polarization sensitivity, we construct a spatial spectrum to estimate the azimuth. After that, a spectrum in the polarization domain can be constructed based on the obtained DOA estimation. Furthermore, the angles of the signals are reestimated to improve the accuracy of DOA estimation by using all the output of the array. Finally, Monte Carlo simulations are carried out to demonstrate the excellent performance of the algorithm.

\section{Appendix}

\section{A. The Dual-Polarized Forward and Backward Smoothing Algorithm}

The dual-polarized forward and backward smoothing algorithm mentioned in this article is based on the traditional forward and backward smoothing algorithm. According to the previous analysis, the dual-polarized cylindrical 
conformal array cannot be directly smoothed. It needs to be projected into a dual-polarized rectangular planar array first and then smoothed to achieve decoherence. The steering vector of the projected dual-polarized rectangular array can be as follows:

$$
\widetilde{\mathbf{a}}_{v}(\theta, \phi)=\mathbf{a}_{z}(\theta) \otimes \widetilde{\mathbf{a}}_{p}(\theta, \phi),
$$

where

$$
\left\{\begin{array}{l}
\mathbf{a}_{z}(\theta)=\left[1, \beta_{z \mathrm{i}}, \beta_{z \mathrm{i}}^{2} \ldots, \beta_{z \mathrm{i}}^{N-1}\right]^{\mathrm{T}} \\
\widetilde{\mathbf{a}}_{p}(\theta, \phi)=\left[1, \beta_{p i}, \beta_{p i}{ }^{2} \ldots, \beta_{p i}{ }^{N-1}\right]^{\mathrm{T}} \otimes \mathbf{I}_{2} .
\end{array}\right.
$$

Thus, if there are $K$ signals in the space, the output signal of the dual-polarized rectangular planar array is shown as follows:

$$
\mathbf{x}_{v}(t)=\tilde{\mathbf{A}}_{v} \mathbf{P s}(\mathrm{t})+\mathbf{n}(\mathrm{t}) .
$$

Since we have already obtained the estimation of elevation, the purpose of smoothing is to estimate azimuth. Thus, matrix $\mathbf{D}$ is shown as follows:

$$
\mathbf{D}=\left[\begin{array}{ccccc}
\mathbf{J}_{\mathrm{D}} & \mathbf{0} & \cdots & \mathbf{0} & \mathbf{0} \\
\mathbf{0} & \mathbf{J}_{\mathrm{D}} & \cdots & \mathbf{0} & \mathbf{0} \\
\vdots & \vdots & \ddots & \vdots & \vdots \\
\mathbf{0} & \mathbf{0} & \cdots & \mathbf{J}_{\mathrm{D}} & \mathbf{0} \\
\mathbf{0} & \mathbf{0} & \cdots & \mathbf{0} & \mathbf{J}_{\mathrm{D}}
\end{array}\right] .
$$

In order to ensure that the smoothing is taken between copolarized components, the cross-polarized components do not interfere with each other. The matrix $\mathbf{J}_{\mathrm{D}}$ is defined as follows:

$$
\mathbf{J}_{\mathrm{D}}=\left[\begin{array}{ccccccccc}
0 & 0 & 0 & 0 & \cdots & 0 & 0 & 1 & 0 \\
0 & 0 & 0 & 0 & \cdots & 0 & 0 & 0 & 1 \\
0 & 0 & 0 & 0 & \cdots & 1 & 0 & 0 & 0 \\
0 & 0 & 0 & 0 & \cdots & 0 & 1 & 0 & 0 \\
\vdots & \vdots & \vdots & \vdots & \ddots & \vdots & \vdots & \vdots & \vdots \\
0 & 0 & 1 & 0 & \ddots & 0 & 0 & 0 & 0 \\
0 & 0 & 0 & 1 & \cdots & 0 & 0 & 0 & 0 \\
1 & 0 & 0 & 0 & \cdots & 0 & 0 & 0 & 0 \\
0 & 1 & 0 & 0 & \cdots & 0 & 0 & 0 & 0
\end{array}\right] .
$$

Assuming that there is only one signal, the following equation can be obtained:

$$
\begin{aligned}
& \mathbf{D} \widetilde{\mathbf{a}}_{v}^{*}=\left[\begin{array}{ccccc}
\mathbf{J}_{\mathrm{D}} & \mathbf{0} & \cdots & \mathbf{0} & \mathbf{0} \\
\mathbf{0} & \mathbf{J}_{\mathrm{D}} & \cdots & \mathbf{0} & \mathbf{0} \\
\vdots & \vdots & \ddots & \vdots & \vdots \\
\mathbf{0} & \mathbf{0} & \cdots & \mathbf{J}_{\mathrm{D}} & \mathbf{0} \\
\mathbf{0} & \mathbf{0} & \cdots & \mathbf{0} & \mathbf{J}_{\mathrm{D}}
\end{array}\right]\left[\begin{array}{c}
\widetilde{\mathbf{a}}_{p} \\
\beta_{z} \widetilde{\mathbf{a}}_{p} \\
\vdots \\
\beta_{z}^{N-1} \widetilde{\mathbf{a}}_{p}
\end{array}\right]^{*}=\left[\begin{array}{c}
\mathbf{J}_{\mathrm{D}} \widetilde{\mathbf{a}}_{p}^{*} \\
\mathbf{J}_{\mathrm{D}} \beta_{z} \widetilde{\mathbf{a}}_{p}^{*} \\
\vdots \\
\mathbf{J}_{\mathrm{D}} \beta_{z}^{N-1} \widetilde{\mathbf{a}}_{p}^{*}
\end{array}\right] \\
& \mathbf{J}_{\mathrm{D}} \widetilde{\mathbf{a}}=\left[\begin{array}{ccccccccc}
0 & 0 & 0 & 0 & \cdots & 0 & 0 & 1 & 0 \\
0 & 0 & 0 & 0 & \cdots & 0 & 0 & 0 & 1 \\
0 & 0 & 0 & 0 & \cdots & 1 & 0 & 0 & 0 \\
0 & 0 & 0 & 0 & \cdots & 0 & 1 & 0 & 0 \\
\vdots & \vdots & \vdots & \vdots & \ddots & \vdots & \vdots & \vdots & \vdots \\
0 & 0 & 1 & 0 & \ddots & 0 & 0 & 0 & 0 \\
0 & 0 & 0 & 1 & \cdots & 0 & 0 & 0 & 0 \\
1 & 0 & 0 & 0 & \cdots & 0 & 0 & 0 & 0 \\
0 & 1 & 0 & 0 & \cdots & 0 & 0 & 0 & 0
\end{array}\right]\left[\begin{array}{cc}
1 & 0 \\
0 & 1 \\
\beta_{p i}^{-1} & 0 \\
0 & \beta_{p i}^{-1} \\
\vdots & \vdots \\
\beta_{p i}^{-(N-1)} & 0 \\
0 & \beta_{p i}^{-(N-1)}
\end{array}\right]_{p} \\
& =\left[\begin{array}{cc}
1 & 0 \\
0 & 1 \\
\beta_{p i} & 0 \\
0 & \beta_{p i} \\
\vdots & \vdots \\
\beta_{p i}^{N-1} & 0 \\
0 & \beta_{p i}^{N-1}
\end{array}\right]\left[\begin{array}{cc}
\beta_{p i}^{-(N-1)} & 0 \\
0 & \beta_{p i}^{-(N-1)}
\end{array}\right] \\
& =\tilde{\mathbf{a}}_{p} \boldsymbol{\Psi}_{i} \text {. }
\end{aligned}
$$

After the above analysis, when there are $K$ signals in the space, the following equation can be obtained:

$$
\mathbf{D} \widetilde{\mathbf{A}}_{v}^{*}=\widetilde{\mathbf{A}}_{v} \Psi,
$$

$\Psi=\operatorname{diag}\left\{\beta_{p 1}^{-(N-1)}, \beta_{p 1}^{-(N-1)}, \ldots, \beta_{p K}^{-(N-1)}, \beta_{p K}^{-(N-1)}\right\}$. Then, we define two covariance matrices $\mathbf{R}^{f}$ and $\mathbf{R}^{b}$ to achieve smoothing.

$$
\begin{aligned}
\mathbf{R}^{f} & =\mathbf{R}^{b} E\left\{\mathbf{x}_{v}(t)\left[\mathbf{x}_{v}(t)\right]^{\mathrm{H}}\right\} \\
& =\widetilde{\mathbf{A}}_{v} \mathbf{P} \mathbf{R}_{s} \mathbf{P}^{\mathrm{H}} \widetilde{\mathbf{A}}_{v}^{\mathrm{H}}+\sigma^{2} \mathbf{I} \\
& =\mathbf{D}\left(\mathbf{R}^{f}\right)^{*} \mathbf{D}^{\mathrm{H}} \\
& =\mathbf{D}\left(\widetilde{\mathbf{A}}_{v} \mathbf{P} \mathbf{R}_{s} \mathbf{P}^{\mathrm{H}} \widetilde{\mathbf{A}}_{v}^{\mathrm{H}}\right)^{*} \mathbf{D}^{\mathrm{H}}+\sigma^{2} \mathbf{I} \\
& =\widetilde{\mathbf{A}}_{v} \boldsymbol{\Psi} \mathbf{P}^{*} \mathbf{R}_{s}^{*}\left(\mathbf{P}^{\mathrm{H}}\right)^{*} \boldsymbol{\Psi}^{\mathrm{H}} \tilde{\mathbf{A}}_{v}^{\mathrm{H}}+\sigma^{2} \mathbf{I} .
\end{aligned}
$$


The smoothed covariance matrix is shown as follows:

$$
\begin{aligned}
\mathbf{R}^{f b} & =\frac{1}{2}\left(\mathbf{R}^{f}+\mathbf{R}^{b}\right) \\
& =\widetilde{\mathbf{A}}_{v} \mathbf{R}_{s}^{f b} \widetilde{\mathbf{A}}_{v}^{\mathrm{H}}+\sigma^{2} \mathbf{I},
\end{aligned}
$$

where

$$
\begin{aligned}
\mathbf{R}_{s}^{f b} & =\frac{1}{2}\left(\mathbf{P R}_{s} \mathbf{P}^{\mathrm{H}}+\boldsymbol{\Psi} \mathbf{P}^{*} \mathbf{R}_{s}^{*}\left(\mathbf{P}^{\mathrm{H}}\right)^{*} \boldsymbol{\Psi}^{\mathrm{H}}\right) \\
& =\frac{1}{2}\left[\begin{array}{ll}
\mathbf{P} & \boldsymbol{\Psi} \mathbf{P}^{*}
\end{array}\right]\left[\begin{array}{c}
\mathbf{P}^{\mathrm{H}} \\
\left(\mathbf{P}^{\mathrm{H}}\right)^{*} \boldsymbol{\Psi}^{\mathrm{H}}
\end{array}\right] \\
& =\frac{1}{2} \mathbf{C}_{f} \mathbf{C}_{f}^{\mathrm{H}} .
\end{aligned}
$$

For a matrix, its rank is equal to the rank of this matrix multiplied by its own conjugate transpose matrix. Thus, we can see that the rank of $\mathbf{R}_{s}^{f b}$ and the rank of $\mathbf{C}_{f}$ are equal from equation (A.10). From the expression of polarization parameter matrix $\mathbf{P}$ in equation (8) and matrix $\Psi$ in equation (A.7), we can deduce that the ranks of the matrices $\mathbf{P}$ and $\Psi$ are both $K$, and their columns are irrelevant. Thus, $\mathbf{C}_{f}$ is a matrix of rank $2 K$. And $\mathbf{R}_{s}^{f b}$ is a matrix with a rank of $2 K$. The smoothing algorithm has completed the process of restoring the rank from $K$ to $2 K$.

\section{B. The Derivation of CRB}

This section describes the derivation of the Cramer-Rao bound (CRB). The CRB offers a lower bound on the variance of unbiased estimation. We derived the CRB using a dualpolarized cylindrical conformal array to compare the performance of the proposed algorithm [20]. The probability density function (PDF) of the output signal $\mathbf{x}$ can be used to construct the CRB. The PDF is defined by $\mathrm{p}(\mathbf{x} \mid \zeta)$. Define the unknown parameter vector in covariance matrix $\mathbf{R}$ as $\zeta=[\theta, \phi, \gamma, \eta]^{\mathrm{T}}$. Because there are $K$ signal sources, the number of elevation and azimuth and two polarization parameters are both $K$. The entries of the Fisher Information Matrix (FIM) can be calculated by

$$
\mathbf{F}_{i j}=-E\left\{\frac{\partial \ln (\mathrm{p}(\mathbf{x} \mid \zeta))}{\partial \zeta_{i} \partial \zeta_{j}}\right\}
$$

where $i, j=1,2, \ldots, 4 K$. Assume that there are $L$ statistically independent observations of $\mathbf{x}(t)$, PDF can be expressed as follows:

$$
\begin{aligned}
\mathrm{p}(\mathbf{x} \mid \zeta)= & \frac{1}{\left(2 \pi \sigma_{n}^{2}\right)^{L / 2}} \cdot \\
& \left.\exp \left\{-\frac{1}{\sigma_{n}^{2}} \sum_{n=0}^{L-1} \| \mathrm{x} n\right)-\mathbf{C A}(\theta, \phi) \mathbf{P s}(t) \|^{2}\right\} .
\end{aligned}
$$

Combining (A.11) and (A.12), the FIM can be rewritten as follows:

$$
\mathbf{F}_{i, j}=L \cdot S N R \cdot\left(\frac{\partial(\mathbf{C A P})^{\mathrm{H}}}{\partial \zeta_{i}} \frac{\partial \mathbf{C A P}}{\partial \zeta_{j}}+\frac{\partial \mathbf{C A P}}{\partial \zeta_{i}} \frac{\partial(\mathbf{C A P})^{\mathrm{H}}}{\partial \zeta_{j}}\right)
$$

where $S N R=\sigma_{s}^{2} / \sigma_{n}^{2}$ and $E\left[s^{2}(n)\right]=\sigma_{s}^{2}$. The process of obtaining the partial derivatives of the array manifold CAP with respect to the vector $\zeta$ is actually the process of obtaining the partial derivatives of the unknown parameters $\theta, \phi, \gamma, \eta$ separately, as follows:

$$
\begin{gathered}
\frac{\partial \mathbf{C A} \mathbf{P}}{\partial \theta_{i}}=\mathbf{C} \frac{\partial \mathbf{A}}{\partial \theta_{i}} \mathbf{P} \\
\frac{\partial \mathbf{C A} \mathbf{P}}{\partial \phi_{i}}=\mathbf{C} \frac{\partial \mathbf{A}}{\partial \phi_{i}} \mathbf{P} \\
\frac{\partial \mathbf{C A} \mathbf{P}}{\partial \gamma_{i}}=\mathbf{C A} \frac{\partial \mathbf{P}}{\partial \gamma_{i}} \\
\frac{\partial \mathbf{C A} \mathbf{P}}{\partial \eta_{i}}=\mathbf{C A} \frac{\partial \mathbf{P}}{\partial \eta_{i}} .
\end{gathered}
$$

Therefore, the whole FIM is a $4 K \times 4 K$ matrix, which can be expressed as follows:

$$
\mathbf{F}=\left[\begin{array}{llll}
\mathbf{F}_{\theta \theta} & \mathbf{F}_{\theta \phi} & \mathbf{F}_{\theta \gamma} & \mathbf{F}_{\theta \eta} \\
\mathbf{F}_{\phi \theta} & \mathbf{F}_{\phi \phi} & \mathbf{F}_{\phi \gamma} & \mathbf{F}_{\phi \eta} \\
\mathbf{F}_{\gamma \theta} & \mathbf{F}_{\gamma \phi} & \mathbf{F}_{\gamma \gamma} & \mathbf{F}_{\gamma \eta} \\
\mathbf{F}_{\eta \theta} & \mathbf{F}_{\eta \phi} & \mathbf{F}_{\eta \gamma} & \mathbf{F}_{\eta \eta}
\end{array}\right] .
$$

Defining $\mathbf{G}=\mathbf{F}^{-1}$, the $\mathrm{CRB}$ can be expressed as follows:

$$
\begin{aligned}
\mathrm{CRB}_{\theta} & =\sqrt{\frac{1}{2 K} \sum_{i=1}^{K} \mathbf{G}_{i i}} \\
\mathrm{CRB}_{\phi} & =\sqrt{\frac{1}{2 K} \sum_{i=K+1}^{2 K} \mathbf{G}_{i i}} \\
\mathrm{CRB}_{\gamma} & =\sqrt{\frac{1}{2 K} \sum_{i=2 K+1}^{3 K} \mathbf{G}_{i i}} \\
\mathrm{CRB}_{\eta} & =\sqrt{\frac{1}{2 K} \sum_{i=3 K+1}^{4 K} \mathbf{G}_{i i} .}
\end{aligned}
$$

$\mathrm{CRB}_{\theta}$ and $\mathrm{CRB}_{\phi}$ are the Cramer-Rao bound for DOA. $\mathrm{CRB}_{\gamma}$ and $\mathrm{CRB}_{\eta}$ are the Cramer-Rao bound for polarization sensitivity.

\section{Data Availability}

The data used to support the findings of this study are available from the corresponding author upon request. 


\section{Conflicts of Interest}

The authors declare that they have no conflicts of interest.

\section{References}

[1] X. Lan, Y. Li, and Y. Chen, "A novel 2D DOA estimation via tensor modeling for cylindrical conformal array," in Proceedings of the 2017 9th International Conference on Wireless Communications and Signal Processing (WCSP), pp. 1-6, Nanjing, China, October 2017.

[2] Z. Ye and C. Liu, "2-D DOA estimation in the presence of mutual coupling," IEEE Transactions on Antennas and Propagation, vol. 56, no. 10, pp. 3150-3158, 2008.

[3] M. Wang, X. Ma, S. Yan, and C. Hao, "An autocalibration algorithm for uniform circular array with unknown mutual coupling," IEEE Antennas and Wireless Propagation Letters, vol. 15, pp. 12-15, 2016.

[4] P. Yang, F. Yang, and Z. Nie, "DOA Estimation Using Music Algorithm on a Cylinderical Conformal Array," in Proceedings of the 2007 IEEE Antennas and Propagation Society International Symposium, pp. 5299-5302, June, 2007.

[5] A. G. Jaffer, "Sparse mutual coupling matrix and sensor gain/ phase estimation for array auto-calibration," in Proceedings of the 2002 IEEE Radar Conference, pp. 294-297, IEEE Cat. No.02CH37322), Long Beach, CA, USA, April, 2002.

[6] A. Bazzi, D. T. M. Slock, and L. Meilhac, "Online angle of arrival estimation in the presence of mutual coupling," in Proceedings of the 2016 IEEE Statistical Signal Processing Workshop (SSP), pp. 1-4, Palma de Mallorca, Spain, Auguest, 2016.

[7] C. Liu and S. Yin, "Direction finding for conformal array in the presence of array perturbations," in Proceedings of the 2015 2nd International Conference on Information Science and Control Engineering, pp. 707-711, Shanghai, China, April, 2015.

[8] M. Wang, Z. Wang, and Z. Cheng, "Joint calibration of mutual coupling and channel gain/phase inconsistency using a near-field auxiliary source," in Proceedings of the 2016 IEEE 13th International Conference on Signal Processing (ICSP), pp. 394-398, Chengdu, China, November, 2016.

[9] C. Liu, S. Xiang, L. Xu, and Z. Fang, "Polarization and DOA estimation based on dual-polarized conformal array," International Journal of Antennas and Propagation, vol. 2019, Article ID 8907685, 9 pages, 2019.

[10] K. P. Ray, R. K. Kulkarni, and B. Kasyap Ramkrishnan, "DOA estimation in a multipath environment using covariance differencing and iterative Forward and Backward spatial smoothing," in Proceedings of the 2008 International Conference on Recent Advances in Microwave Theory and Applications, pp. 794-796, Jaipur, India, November, 2008.

[11] A. Richter, F. Belloni, and V. Koivunen, "DoA and polarization estimation using arbitrary polarimetric array configurations," in Proceedings of the Fourth IEEE Workshop on Sensor Array and Multichannel Processing, pp. 55-59, Waltham, MA, USA, July, 2006.

[12] M. Costa, A. Richter, and V. Koivunen, "DoA and polarization estimation for arbitrary array configurations," IEEE Transactions on Signal Processing, vol. 60, no. 5, pp. 23302343, 2012.

[13] Z. s. Qi, Y. Guo, B. h. Wang, Y. Guo, and B. h. Wang, Blind direction-of-arrival estimation algorithm for conformal array antenna with respect to polarisation diversity," IET
Microwaves, Antennas \& Propagation, vol. 5, no. 4, pp. 433442, 2011.

[14] C. Liu, Z. Ding, and X. Liu, "Pattern Synthesis for Conformal Arrays with Dual Polarized Antenna Elements," in Proceedings of the 2014 7th International Congress on Image and Signal Processing, pp. 968-973, Dalian, China, October, 2014.

[15] Y. Zhang, C. Liu, X. Xu, and Z. Ye, "Direction of Arrival Estimation in the Presence of Array Perturbations," in Proceedings of the 2010 2nd International Conference on Education Technology and Computer, Shanghai, China, June, 2010.

[16] Z. Zhongfu Ye, J. Jisheng Dai, X. Xiaopei Wu, and X. Wu, "DOA estimation for uniform linear array with mutual coupling," IEEE Transactions on Aerospace and Electronic Systems, vol. 45, no. 1, pp. 280-288, Jan. 2009.

[17] C. Shu and Y. Liu, "An improved forward/backward spatial smoothing root-MUSIC algorithm based on signal decorrelation," in Proceedings of the IEEE Workshop on Advanced Research and Technology in Industry Applications (WARTIA), pp. 1252-1255, Ontario, Canada, September, 2014.

[18] R. Boyer and G. Bouleux, "Oblique projections for directionof-arrival estimation with prior knowledge," IEEE Transactions on Signal Processing, vol. 56, no. 4, pp. 1374-1387, 2008.

[19] S. Mohammadi, A. Ghani, and S. H. Sedighy, "Direction-ofArrival estimation in conformal microstrip patch array antenna," IEEE Transactions on Antennas and Propagation, vol. 66, no. 1, pp. 511-515, 2018.

[20] S. van der Tol and S. J. Wijnholds, "CRB analysis of the impact of unknown receiver noise on phased array calibration," in Proceedings of the Fourth IEEE Workshop on Sensor Array and Multichannel Processing, pp. 185-189, Waltham, MA, USA, July, 2006. 